Hospital Manuel Arriarin.

\title{
MENINGITIS A PFEIFFER
}

Con especial referencia al tratamiento asociado penicilina-sulfa.

Por los Dres. JUL10 MENEGHELlo y GLADYS SUDZUKI H.

Desde el año 1945 hasta la fecha, en el Servicio de Infecciosos del Hospital Manuel Arriarán, se han hospitalizado 8 casos de meningitis a Bacilo de Pfeiffer. Los 3 primeros fueron tratados cen el método convencional con sulfas y cotrespondian a niños cuyas edades eran inferiores a 9 meses $y$ todes fallecieron en plazos variables. Los otros 5 niños, que son el motivo de esta presentación, fueron tratados con penicilina asociada a sulfas. De éstos. 3 mejoraron y 2 fallecieron.

Llamamos, en primer lugar. Ia atención sobre la relativa rareza de la meningitis a Pfeiffer en nuestro Hospital, ya que en el mismo plazo bemos tenido la oportunidad de atender 23 casos de meningitis a neumococo. Sabemos que autores norteamericanos (3), argentinos (14), uruguayos (7) y aun en nuestro mismo medio, pero en otros hospitales de Santiago, sostienen $e^{1}$ aumento creciente de la meningitis a Pfeiffer. ocupando ésta el primer lugar de frecuencia entre las meningitis purulentas. Esta relativa rareza en nuestro medio nos ha itupedido presentar, en esta ocasión, un número mayor de enfermos tratados con la asociación penicilina-sulfa, la cual, por razones que expondremos, no nos parece terapia ilógica en afección tan grave.

La meningitis a Pfeiffer en el lactante menor ha sido casi invariablemente fatal en todo el mundo, con excepción da los casos tratados con suero específico de conejo, donde aun 
la misma Dra. Alexander (1) ha logrado sólo disminuir la mortalidad con este tratamiento asociado al sulfa a un $75 \%$, en los menores de 7 meses.

Sabemos que en la literatura hay muchos casos aislados que mejoraron con solo sulfas, de modo que estamos enterados que el éxito obtenido en los enfermitos que comunicamos, no está exento de críticas. Sin embargo, como se verá, todos nuestros enfermos llegaron al Hospital con varios días de evolución y con glucosa por debajo de la cifra que se acepta como recuperables con sólo sulfas. Llama la atención, por otra parte, que especialmente autcres uruguayos, han venido comunicando éxitos con el tratamiento penicilina-sulfa, sin dar ura explicación satisfactoria, ya que hasta ese entonces el bacilo de Pfeiffer aparecía como uno de lós gérmenes penicilinoresistentes. En estas condiciones aparece que el uso de la penicilina asociada a la sulfadiazina (bacteriostasis) no es un ensayo ilógico en una afección, en la cual, por lo menos en nuestro medio, no tenemos por ahora la posibilidad del suero de Alexander.

A continuación expondremos con detalle las 5 observaciones que motivan esta comunicación.

\section{CASO N 1. - Obs, $12197 / 46$. - Edad: 9 meses.}

Antecedentes bereditarios. - Padte de 28 años de edad, con serologia (-) este año. Madre de 19 años. słana. con Reacción Kahn (-) hace 4 meses atrás. Primer hijo.

No hay antecedentes de tuberculosis familiar.

Antecedentes persanales. - Nació de término, en parto normal. Alimentado a pecho exclusivo 2 meses. desfues de los cualos agrega milo en agua corso relleno. Recibe esta alimentación hasta los 5 mesøs, en que reemplaza 2 veces el pecho por leche de vaca (4 cucharadas de leche por taza de agua con una cucbaradita de Milo). A los 6 meses agrega una sopa de posta, no habiendo posteriormente ningún otro cambio en la alimentación hasta su hospitalizactión.

Enfermedades anteriores: Coqueluche a los ? meses de edad,

Enfermedad actual. - Se inicia 8 dias antes del jngteso $\cos \operatorname{tos} \mathrm{y}$ coti7.a. agregándose posteriormente ficbre, decaimiento y vómitos a repetición. Ingresa al Hospical el dia 23-VII-1946. sin haber sido sometido a ningún tratamiento en su casa.

El examen físico de ingreso demuestra una niña grave distrófica, páliła. febril, con pulso pequeño, encontrándose como único signo meníngeo la tensióil de la fontanela. El seasorio estaba lúcido. aunque tendia a la somnolen- 
cia. La panción lumbar rẹeló líquido purulento, a presión, con Fandy

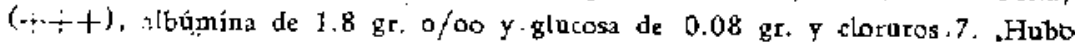
desatrollo de Bacilo de Pfeiffer. tipo $B$ al eultivo, del que demostró ser resistentc a la penicilina, prto no se especificó el númeto de onidades a que fué sonietido.

La evolacion del cuadro meninge fuć farorable. El único signo meningeo que presentó lo enfermita, o sea, la tentión de la fontanela desaparació a la semana de iniciada la enfermizdad, junto con la sommolencia del ingreso. La curva térnica, alta $\mathfrak{c}$ irregular durante la primera semana. se hizo nocmal durante 3 dias y luego osciló alrededor de 37.5, para normalizarse definitivamente al $18^{\circ}$ dia de estada. El liquido oéfalo-raquideo fué turbio $y$ opalescente los ptimeros 19 dias de estada. haciéndose posteriomente claro y normalizandose a kos 26 dias de estar la niña hospitalizada. El germen sólo a evidenció en el primer examen de líquido céfalo-raquideo. Las cifras de glucosi de $0.04 \mathrm{gr}$. al ingreso. Fueron tapidamente elevándose hasta alcanzar a 0.40 gr. o/oo después del $12^{\prime \prime}$ dia. La albuminoraquia alcanzó cifras hasta de $1.8 \mathrm{ge}, 0 / 00$. El estado fsiquico, pasada la primata semana de la somno. lencia, fué muy buerio, estando la chica onimada y alegre durante casi toda sa estida.

En la convalecencia de su meningitis presenta una otitis derecha. que obliga a bacet und paracentesis. Pasa la otitis $y$ nuevamente experimenta febricolas, que haltan explicación en una piuria, que se trató con sulfatiazol. hasta obtener la normalización de sus exámencs de otina.

Tratamienco. - Sulfatiazol oral, a razón de $0.38 \mathrm{gr}$, por kilo de peso por 36 dias, lo que da un total de 105 ges. Finicilina intrataqujda on aft ritno de 24 horas y con dosis de $10,000 \mathrm{U}$. excepto la primesa, que fuci de 5,000 U., con un total de $255.000 \mathrm{U}$, en 26 punciones.

La medicación pericilinica intratecal se continuó por dos dias más despues de haberse normalizado el liquido céfalo raquideo, continuándose con el sulfatiazot por 17 dias mis, Estuvo hospitalizada 63 dias.

E1 dia 16, XI, o sea, al mes $y$ medio de retitada del hospital. la chica scude a controt, encontrándose en perfectas condiciones.

Resumen. - Niña de 9 meses de edad, que ingresa al Hospital con un cuadro meníngeo iniciado 8 días antes, sin haber sido sometida a tratamiento.

A su ingreso se comprueba una enfermita grave con meningitis a bacilo de Pfeiffer; líquido céfalo-raquídeo purulento y glucorraquia de $0,08 \mathrm{gr} .0 / 00$. Se instituye inmediatamente la terapia combinada penicilina-sulfa, bajo cuya acción se asiste a la curación de la niña en 26 dias. 
CASO No 2. - Obs. N" 20003/46. - Edad: 8 meses.

Antecedentes bereditarios. - Padres vivos, sanos, con Reacción de Kabn (-) ambos hace 2 años.

Dos hijos viros y saoos.

Sin antecedentes de tuberculosis familiar.

Antecedenules personales. - Nacido de término, en parto normal, pesó al nacer 3 kilos. Alimentado a pacho exclusivo basta su bospitalización.

Enfermedades anteriores: Desdé su-nacimiento basta la fech d coriza seroso. Bronconeumonia a los 3 meses de edad.

Enfermedad actual, - Ingresa al hospital el dia 23-V, muy grave, con una poliomielitis anterior aguda con compromiso bulbar, iniciada 4 dias antis de su hospitalización $y$ que comprometia las 4 extremidades. El líqnito céfalo-racaideo, a sa ingreso, fué el siguiente: Aspecto claro: Pandy $(++)$ : albúmina. 30; glucosa, 0,70 gr. o/oo; clornros. 7; leucocitos, 40 por mm. Posteriormente, al $7^{\circ}$ dia de estada, se descubre en la niña noa reacción de Kala $(+++)$, e investigada la sangte en la madre se encuentra en ella un Kahn $(+t+)$. er vista de lo sual, unido a los signos de hepato y esplenomagalia y coriza, que presenta la enfermita, permiten hacer el diagnóstico de una lús en evolución, que se trata con penicilisa intramuscular, a razón de $10,000 \mathrm{U}$. calda 3 horas pot 8 dias, colocando un total de $490,000 \mathrm{U}$.

A los 17 dias de estada. la curva térnica se hace norma!, coincidiendo csto con ona mejotia evidente del estado general. $E_{n}$ estas condiciones se hace al 19" día una punción lumbar, con el fin de descartar una lúes nerviasa, eriontrándose un líquido céfalo raquídeo con Fandy $(t)$ débil; albúminia de 0.24 gr. o/oo: glucosa de $0.58 \mathrm{gt}$. o/00: cloruros. 7 ;eucocitos. 1,2 for mm. Pasa la chica dos dias afebriles y bruscamente, al $20^{\circ}$ día. la temperztara sube a 39 grados. aparece intranquilidad. empieza a rechazar alimento. Hay al examen físico coriza y faringe roja, lo que sirvió para explicar el cuadro febril al principio pero a los 8 dias de estar en estas condiciones. aparece tensión de la fontanela y rigider. de la nuca. to que planteó la posibilidad de un cuadro meníngeo. Se hace una punción lambar, que da salida a liquito cifaloraquideo purulente, ton Pandy $(+++)$; albúmita, 0,80 ; slucosa, $0.05 \mathrm{gr} . \%$ oo, encontrándose bacilos de Pfeiffer al cultivo, el que no volvió a aparecer en exámenes posteriores. Se inicia el tratamiento pénicilina-sulfa $y$ el liquido céfalo-raquideo se hace opalescente $y$ se aclara totalmente al $17^{\text {n }}$ día de iniciada la enfermedad, normalizándose del todo a los 21 días de iniciado el cuadro meníngeo. La glucosa experimentó grandes variaciones, llegando. en ocasiones, a $0,04 \mathrm{gr}$. O/oo en el periodo más culminante de la enfermedad.

En cuanto a sus signos meningeos nanca hubo Kernig ni Brudzinsky, sindo de sus signos meningeos la tensión de la fontanela la primera en desaparecet. Llama la atención de que los signos meningeos desaparecieron del todo como a los 5 dias después de haberạ normalizado el liquido céfatoraquideo. 
La temperatura se mantuvo alta, llegando hasta 39,5 por 8 dias, al sabo de los calales se normalizó del todo.

Controles posteriores al mes y 40 dias después de iniciado el cuadro meningeo demostraton liquidos céfalo-raquideos normales, siendo el último examen el siguiente: Aspecto claro; Fandy (-); albúmina, $0,10 \mathrm{gr} .0 / \infty$; glucosa, $0.50 \mathrm{gr}$. $0 / 00$; cultivo (-).

Posteriormente, la chica siguió hospitalizada, curándose su lúes. con intensas complicaciones óseas. motivo pot el cual lleva 226 dias en ef servicio. Esta larga permaneacia ha servido para controlar Ja curación total de su meningitis a Pfeiffer.

Tratamiento. Estuvo 4 dias sin tratarse, cuando aun no se bacia el diagnóstico de meningitis, al cabo de los cuales se inicia una cura con solfatiazol, a razón de $0,17 \mathrm{gr}$, por kilo de peso durante los 3 primeros días, luego se eleva la dosis a $0.35 \mathrm{gr}$. por kilo de peso por otros 3 días, cambiando a esta altura el sulfatiazol por 1 a sulfadiazina a iguales dosis por 19 dias más. Posteriormente se eleva la dosis a $0,50 \mathrm{gr}$. por kilo de peso por otros 12 días, disminuyéndola luego a $0.35 \mathrm{gr}$. por 9 días y a $0,17 \mathrm{gr}$. par kílo de peso los últimos? dias, dz modo que recibió un total de 72,38 grs. de sulfas en 43 dias.

Además del tratamiento por vía oral, se reforzó la cantidad de droga con 21 empleo de diaseptil endovenoso (sulfadiazino sódica), a razón de un cuarto de anpolfeta cada 6 horas durante 15 dias, en un total de 15 grs.

Simoltáneamente con la iniciación del tratamiento con sulfas, se empleó penicilina intra-raquidea, a razón de $10,000 \mathrm{U}$, diarias durante 21 días, co: locardo un total de $210,000 \mathrm{U}$.

A la primera inyección de penicilina intratecal se sacedió un descenso dz la temperatura a la normal. Se deja de colocar ésta y vuelve a subir la temperatura a 38,5 . bajando otra vez a la normal al reiniciar la terapia intratreal.

La concentración de sulfadiazina más alta en la sungre fué de 14.8 mets. \%.

La concentración más alta de sulfadiazina en el líquido cétalo-raquideo fué de 10.8 migrs. \%

La sangre ile este enfermito demostró un poder bemobaccericida màs que regajar.

La medicación de penicilina intratecal se continuó por espacio de 10 dias mís. aespués de haberse normalizado el liquido céfalo-raquídeo, y la sulfadia zina oral por 21 dias más.

Resumen. - Niña de 8 meses que, estando hospitalizada por una poliomielitis anterior aguda, hace en el Servicio una meningitis a Bacilo de Pfeiffer, la que no fué diagnosticada hasta el $8^{\circ}$ día del comienzo aparente de su enfermedad. 
El líquido céfalo-raquídeo extraido antes de iniciado el tratamiento da una glucorraquia de $0.05 \mathrm{gr}$. $\% / 0$, siendo grave el estado general de la chica, ya que había dos enfermedades evolucicnando al mismo tiempo. Se instituye la terapia combinada sulfa-penicilina, bajo cuya acción se asiste a la curazión total del enfermo en 22 días. Lleva la niña alrededor de ó meses de observación y está sana de su meningitis, sin secuelas aparentes.

\section{CASO No 3. - Obs. N: 12127/46. Edad: 4 meses.}

Antecedentes hereditarios.- Padñ de 31 años sano, con secologia (-) en 1945. Madre de 27 años de edad, tratada de lúes por espacio de 2 años. basta I940. en que obtiene serología (-), después de la cual ha tenito hast. la fecha 4 niños sanos con reacción de Kaha (-) todos. Antes de su tratamiento tuvo dos mortinatos.

Sin antecedentes de tuberculosis familiat.

Antocedentes personales. - Nacido de término, en parto normal. Nació en su casa. por la que ignoran el peso de nacimiento. Alimentada a pecho exclusivo sólo 4 días, siguiende. posteriormente, hasta la facha, con pectro 3 veces y 3 mumaderas de leche Nido al dia 12 cuchataditas the leche para 5 cusharadas de agua.

Énfermedades anteriores: No acusa.

Enfermedad acapl. - Se inicia el dia 30-VII-1946. cinco dias antes det ingreso. enn fiebre, cansancio, llanto y malestar geneial. agregúndose vómitos dos dias antes de su hospitalización. A los dos dias de haberse inicizdo la enfermedad es vista por médico particular, quien receta sulfatiazol, alcanzando a tomar 2.25 grs. antes de bospitalizarse. Como no se observara mejoría ningana del cuadro. es traida a la Fosta Infantil del Hospital Arriarán cl día 3-VIII.

El examen fisico de ingreso demuestra una niña grave, pálida. raquítica, excitada, con facies de temor. Febril. con fontaneia tensa acompañada de discreta rigider de la nuca. La punción lumbar demuestra líquido turbio. con Pandy ( + + + ), albúmina de 1 gr. o/oo, glucosa de 0.25 ge. o/00; cloruros. 6 . E: cxamen microscópico revela abundantes glóbulos blancos destruidos, no obsertándose gérmenes. Hay 280 lencocitos por min. 3 con $80 \%$ de polinucleares y $20 \%$ de linfocitos. El bacilo de Pfeiffer sólo vino a evidenciarse al $10^{\circ}$ dia de estada. A los 4 días de estar en el Servicio presenta pasajeramente temblor de las extremidades. Aparece tambièn el Kernig. el que se hace (-) al $20^{\circ}$ dia. La curva febrit, en general, fué baja, haciéndose normal a los 9 dias de estada. continuardo en estas condiciones 13 dias, pata volver a subir hasta 38 grados detance 6 días, normalizándose nuevamente hasta la elevación final; 5 dias antes de la thuette. dias que sobrepasó los 40 grados.

A los 32 dias de estada desaparecieron totalmente sus signos meningeos $y$ ₹l liquida céfalo-raquídeo es claro. con albúmina de $0.10 \mathrm{gr}$. $/ 00$; glucosa de 
0,58 gr.; leucocitos, 4 por mm.3. Cultivo (-). Después de este último liquido céfạlo-raquídeo normal, permance la enfermita en muy buenas condicioges generates. por 28 dias, estando en este lapso completamente afebril y sin ringứn signo moningeo, habiendo subido en este lapso. 780 grs. de peso. A pesiar de estar aparentemente sana de su meningitis, insistimos in continuar la terayia combinada durante 14 dias más. En este periodo se practicaron 5 puncioues de control, revelando todos los exámenes de líquido cifalo-taquídeo, cifras nurmales te glucosa $y$ de albúmina; $y$ sólo ex̄asos elementos telulares. Suspendida la penicilina intra-raquidea, se continuó 16 dias más con sulfadiazina oral, al cabo de los cuales, un uiltimo liquido céfalo-raquideo fú completamente normal. Justo a la semanx siguierite de suspendida la dtoga cuando ya pensábamos darke el alta, sobreviene una agravación brusca e intensa de1 enfermito. que duró 4 áias, con fiebre alta hasta 41 grados, gran palidez de la piel, hipertonia de las extremidades, rigidey de la nuca, presentando crisis convulsiva en las vitimas heras. Fraslece a los 64 dias de estacia. La punción lumbar hecha en escos dias, dió líquido parmento con desarrollo de reumobacilo de Friediander, habiendo descendido la glucosa a $0.02 \mathrm{gr}$. o/oo.

Desgraciadamente no se hizo autopsia y no sabemos hasta qué panto la chica conó de su meningitis a Pfeiffic. ya que su cpisodio final se debió a oiro getmen.

Tracamienro. - Se empleó sulfadiazina oral a razón de $0,35 \mathrm{gr}$. por kilo de peso los primems 26 dias, subiendo posteriormente la dosis a 0.50 gr. por kilo de peso por 21 dias más, luego se disminuye a $0,35 \mathrm{gr}$. por kilo de peso por 8 dias. Total: 79.86 grs. de sulfadiazina en 49 dias.

Simultáneamarte con la salfa oral se administró penicilina intra-raquidea a razón de $10,000 \mathrm{U}$. diarias por 14 días. subiendo después a $15,000 \mathrm{U}$. diarias por otros 7 dias y finalmente se aumenta a $20.000 \mathrm{U}$. diarias por 11 días, colocindo un total de $460,000 \mathrm{U}$. en 32 dias.

Usamos también la penicilina por via intramuscular, $5.000 \mathrm{U}$. cada 3 hozas. en un total de $905.000 \mathrm{U}$. en 24 horas.

La concentración máxima de sulfadiazina encontrada en $\mathrm{la}$ sangre faé de 14.3 mgrs. o/ 00 , alcanzando 1 del líquido céfalo-raquideo a 8.5 grs. o/oo.

La medicación penicilínica intra-raquídea se continuó por 14 días más dejpués de haberse obtenido la normatización del liquido céfalo-raquideo, continzándose con snlfadizzina oral pot 16 dias más.

La tipificación del germen dió bacilo de Pfeiffer tipo B resistente a la panicitina, sin especificarse a qué concentraciones.

Resumen. - Lactante de 4 meses de edad, distrófico, que ingresa al Servicio de Urgencia del Hospital Arriarán, con un cuadro meníngeo iniciado 5 dias antes. habiendo tomado $2.25 \mathrm{grs}$. de sulfatiazol en los 3 últimos dias. En tr cepción es catalogada como una enfermita grave. El priner 
líquido céfalo-raquídeo, extraído en la Posta Infantil. es pur rulento, con glucorraquia de $0,25 \mathrm{gr}$. $0 / 00$, habiendo desarrcllo de Bacilos de Pfeiffer al cultivo. Se instituye terapia $\mathrm{com}^{-}$ binada penicilina-sulfa, bajo cuya acción se asiste a la regrésión total de su meningitis al cabo de 32 días, sobreviniendo un período de normalidad, que dura 28 días, al cabo de lus cuales presenta una meningitis gravisima a neumobacilo de Friedlander y que la conduce a la muerte en 5 días.

CASOO $\mathrm{N}^{0}$ 4. - Obs. $\mathrm{N}^{\mathrm{V}} 18807 / 40$. $\rightarrow$ Edad: 6 ineses.

Antecedences beredicarios. - Madre de 34 año: de edad, sana. coa szrología negativa hace 4 años. Fadre de 29 años. sano. con Reactión de Kahn (-) hace un mes atrás.

Tres hijos vivos y sanos.

En 1928 fallecio la abarla paterna de tubetialesis pulmonar.

Antecedentes personales. - Nacido de término en parto prolongado. María con asfixia azul. Alimentado a pecho paxeluxivo hasta la fecha. Dentición a tos 6 meses.

Enfermedades anteriores: Ninguna.

Enfermedad accusl. - Se inicia en formas beusca el dia $12 \cdot[\mathrm{V}-1946$. can decaimiento general $y$ fiebre. Al día siguisnte continús intranquilo $y$ en la goclk ipresenta convulsiones tónico-clónicas. que comienzan es el miẹmbro sapering izadierdo. generalizándose después. Esre cuadro duró más o menos 15 . minuyos. cayendo después el niño en estado de sopor. condición en que llega a la pasta lnfantil a las 2? horas del dia $13 \mathrm{da}$ abril. sin haberse becho ningun tratamiento anterior a su hospitalización. Tamposo hubo antecedentes de cuadeu respiratorio jrevio.

El examen físico de ingreso tevela un niño grave. fabril. con sınsorio em. burado, rigidez de la nuca pronunciada, kornig positivo. fontanela a tensión. nistagmus ocular, palidez de la piel, reflejos exaitados. La punción lumbar ravela cíquido turbio, purulento con desarrollo de bacilos de Pfeiffer al cứtivo.

A los 4 dias de estar en el Servicio presenta crisis conuulsitas tónico-clónicos de las exiremidades inquierda con desviación conjugada de los globos orulares. La obnubilación sensoria tardó 5 dias en desagarecar del todo. Durance toda si estada presentó vómitos en forma intermitente. A los 7 dias de rstar en el Servicio presenta temblor pasajero da las extremidadis, A los 14 días de estada se produce una petjucña neejoria clinica. sin acompañarse de cambio jacorable en el aspecto del liquido céfalo-raquideo. mejoría que só'o duró 4 dias, al cabo de los cuales el enfermito se agrava iatensanente, piresanta cris's convusivas a repetición, vómitos, inconsiencia y trimo. fallecirado a los 22 dias de estada. Durante toda su permanencia no hubo variación en los signos moningeos encontrados al ingreso. El bacilo de Pfeiffer se encontró en 6 oia- 
siones en el caltivo de líquido géfato-raquídeo. En cuanto a la curva térmica sólo fué normal por 3 días durante su estada de 22 dias:

Tratamiento. - Se empleó el sulfatiazol los 3 primeros dias, en dosis de $0.20 \mathrm{gt}$. por kilo de peso, cambiando posteriormente a sulfadiazina en la misma dosis por otros 3 dias. subiendo 1a dosis a 0.40 gr. por kilo de peso los últimos 14 dias, dando an total de 48,63 grs, de sulfa en 20 días.

Durante los primeros 15 dias de estada usamos diaseptil endovenoso, an gramo diario. colocando un total de $15 \mathrm{grs.}$

Usamos, ademis, la penicilina intra-raquidea a razón de $10,000 \mathrm{U}$. diarias, durante 14 dias, más un día que se colocó $10,000 \mathrm{U}$. dos veces en el día. habiendo colocado un total de $160.000 \mathrm{U}$. en 15 dias.

La concentración máxima de sulfadiazina en la sangre fué de $24 \mathrm{mgrs}$. $\%$. alcanzagdo lá del líquido céfao-raquídeo a $21.8 \mathrm{mgrs}$. \%.

Necropsia. - Leptomeningitis purulenta a bacilo de Pfeiffer. Tumefacejöa turbia del miocardio, hígado y tiñones. Hiperplasia del bazo.

Resumen. - Lactante de 6 meses de edad, que ingresa a la Posta Infantil del Hospital Arriarán con un cuadro meníngeo, iniciado aparentemente un día antes, no habiendo recitido durante este lapso ningún tratamiento. En recepción se comprueba un enfermo en estado de suma gravedad, con una meningitis a Bacilo de Pfeiffer, que se caracterizó por la rebeldía al tratamiento combinado sulfa-penicilina, ya que el bacilo de Pfeiffer reapareció en el líquido céfalo-raquideo en 6 ocasiones, lo que nos hace suponer que la terapia fué insuficiente, dada la gravedad del enfermito.

El enfermo falleció al cabo de 22 días, observándose durante su estada sólo una ligera remisión clínica de unos 4 días de duración, a la semana de estar hospitalizado.

CASO No 5. - Obs. $N^{p} 20003 / 46$. - Edxt 8 meses.

Antecedentes herediórios, - Madre, 26 đños. acusa sombca al pulmón desule bace más o menos 10 meses. estando actuaimente en reposo en el Sagatorjo Lo Franco. Padre. 56 an̆os. aparentemente sano.

Cuatro bijos vivos, uno de ellos cuvo sombra al pulmón hace un año.

El niño fué separado de su madre desde los 3 meses de edad siendo colocado en un hogar sin antecedentzs de tuberculosis, en la Foblación Lo Franco. donde es controlado por el Seguro Obrero hasta la fecha.

Anced:dentes personales. - Nacido de parto notmal, de término, pesó al nacir 2,800 gis. Criado a pecho exclusivo los 3 primeros meses, seguido des. pués con Nestalba hasta los 5 messs, en que se inicia la alimentación con lexhe condensada. controlada en el Seguro Obrero hasta el dia de su bospitalización.

Enfermedades anteriores: Raguitismo a las 6 meses de edad. 
Enfermedad actmal. - Se inicia el día 13 de mayo con fiebre y tos. Consuita al Siguro Obrexo, donde se diagnostica estado gripal, prescribiéndose sulfatiazol, un cnarto de tableta cada 4 horas. Sin embargo, el niño sigue febril. inquieto, gresencando finzintente convulsiones generalizadas, por lo que es lkevado maevamente al conscltorio del Seguro Obrero, donde encuentran signos masingeos claros, por lo cal se ervía inmediatamente al Servicio de Urgencia del Hospical Arriarán. Es hospitalizado enionces al cuarto día de enfermedad, habiendo tomado wa total de 3 grs. de sulfa.

A su ingreso al Hospital se comprueba un enfermito pálido, raquítico. con strisorio lícido, hipertonia muscular generalizada, raya meningea, franca rịidez de la nuca. Brudzinsky positivo, híperestesia cutanes. La punción lumbar da lignido céfalo-raquideo, de aspecto purulento, ton glucorraquia de 0.10 gr. o/oo, encontrándose bacilos de pfeiffer al examen directo y cultivo. Ini. cismos inmediatamente el tratamiento combinado sulfa-penicilina, manteniendo la : ‘rapéntica por 9 dias consecutivos, consiguiendo una gran mejoria del cuadro elínico. Desciende la temperatura a la normal, desaparecen los signos meringros y se obtione a la punción lumbar líquida claro, aséptico. con aumento de la glucosa a $0,40 \mathrm{gr}$ o/oo. En vista de este éxito suspendemos la terapia pinicilinica, continnando con la sulfadiazina oral. El enfermito permanece en buinas condiciones por 4 dís seguidos, volvisndo posteriormente a presentat fietrec, inquietud. excitabilidad anormal, sxaltación de reflejos. dando la punción lumbar nuevamente líquido purulento. babiendo un descenso de ia glucosa a $0.10 \mathrm{gr}$. o/oo. Aparze ruevamente el bacilo de Pfeiffer en el liquido cifato ráquídeo, en vista de lo cual iniciamos nueva cura de penicilina intra-raquidea. masenida durante 12 dias consecntivos, obteniendo nueva remisión del cuadro clitico y acl líquido céfalo-raquideo, volviendo a hacese el liquido claro, aséptici, con cifras dé glucosa elevadas. Suspendemos por terceta vez la penticilina intra-raquidea. con to cual el enfermito decaz nuzvamente. presentando inconscirsia, temblor de las extremidades, fiebre y Brudzinsky positivo. El líquido eificio-raquídeo se bace purulenta, baja esta viz la glucosa a $0.01 \mathrm{gr}$, o/oo. A pincentamos ertonces la culfadiazina oral $(0.50 \mathrm{gr}$. por kilo de peso) $\mathrm{y}$ doblamai la cantidad de penicilina intra-raquidea $(20,000 \mathrm{U}$. diariasi. con lo cual nciamos nueva trejoria clínica del enfermito, acompañada de una franca mejoria del liquido céfalo-raquideo, que se hace nuevamente transparente y aséptico, a la vez que la glucosa ha aumentado, razones for la cual volvemos a susperder la penicilina intra raquidea. § los 3 dias de ello hay nueva agravación de! enfermito, bajando la glucosa del liquido céfalo-raquídeo a $0.03 \mathrm{gr}$. o/oo, lo que coincide con una turva febril más alta quz nunca; alcanzó temperaturas superiors a $40^{\circ}$. Colocamos esta vez penitilina intramusculat y aumentamos la cantidad de penicilina intra-raguides a $40,000 \mathrm{U}$. diarias, sin obtener esta vez éxito alguno. El enfermito sigue agravándose. preseata contracciones fibrilares de los músculos de la cara, temblor de las extremidades y cabeza, intensa hipertenía muscular, rechaza todo alimento, falleciendo finalmente a los. 83 dias de hospitalización. 
Tiarimiento:

Sulfatiazol a tazón de $0.50 \mathrm{gr}$. por kilo de pero, por 8 dias $13.69 \mathrm{grs}$.

Sulfadiazina a razón de $0.50 \mathrm{gr}$, por kilo de peso. por 71 dias 127.5 "

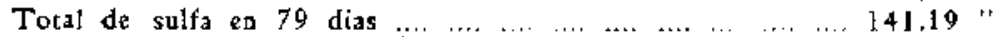

Penicilina intra-raquidea en 48 punciones: $905,000 \mathrm{U}$.

Penicilina intramoscular en 14 dias: $304.000 \mathrm{U}$.

La concentración de sulfadiazita máxima en la saggre fuś de $16.8 \mathrm{mgrs}$. \%̆.

La eoncentración de sulfadiazina máxima en el liquido céfalo-raquideo fué de 12,8 mgrs. r.

Necropsia. - Leptomeningitis purulenia a bacito de Ffeiffer. Intenso piocéfalo interno. Tabicamiento del espacio subdural de la médula a aival de la tercera vértebra dorsal. Trombosis de los senos cavernosos y parcialmente dél sero longitadinal. Tumefacción turbia del miocardio, hígado y tiñones. Hiper. plasia del bazo.

Resumen, - Niño de 9 meses de edad, que ingresa a la Posta Infantil del Hospital Arriarán con estado convulsivo y un cuadro meníngeo iniciado 4 días antes y tratado fuera con sulfatiazol, habiendo tcmado antes del ingreso 3 grs. en 4 dias.

En recepción se comprueba un enfermito grave, con líquido céfalo-raquídeo purulento, con glucorraquia de 0.10 gr. 0,00 y con bacilos de Pfeiffer al examen directo y al cultivo. Se inicia la terapia combinada sulfa-penicilina, bajo cuga acción el enfermo presenta franca remisión de su cuadro meníngeo. seguida de agravación posterior del cuadro apends se suspendía la penicilina intra-raquidea, pese a que se continuaba con la sulfadiazina oral. En efecto, en 3 ocasiones pudimos asistir a remiciones francas del cuadro meníngeo, que aparecian directamente relacionadas con la administración de penicilina. Además, los exáménes de líquido céfalo-raquideo experimentaban, en relación con estas remisiones, índices francos de mejoría con la elevación de la glucosa, la que en ocusiones llegó hasta $0.40 \mathrm{gr}$. o/oo.

El enfermo falleció, pese a nuestra intensa terapia, al cabo de 83 días.

\section{Resultados y comentario.}

Los 5 enfermitos tratados con la asociación penicilinasulfa eran lactantes menores de 10 meses (dos de 9, uno de 6 y otro de 4 meses). De éstos, 3 curaron y 2 fallecieron.

En estos resultados hemos considerado como curado el caso $\mathrm{N}^{\circ} 3$, que, como se señaló en la casuística, se trata de una 
enfermita de 4 meses de edad, tratada en forma intensiva con penicilina intra-raquídea durante 36 dias y con penicilina intramuscular durante 24 días, asociada a la sulfadiazina, 10grándose, al cabo de 32 dias, la mejoría completa del cuadro. En efecto, desde el dia $10^{\circ}$ ya no había fiebre, el niño sè alimentaba en buenas condiciones, su psiquis era normal, sus signos meníngeos fueton disminuyendo en forma lenta, hast3 desaparecer al cabo de 29 días, obteniéndose un líquido céfaloraquídeo normal a los 32 días de estada. A partir de esta fecha, y con el objeto de asegurarse la curación, se prolongó el tratamiento con penicilina intra-raquidea por 14 dias mas $y$ con sulfadiazina oral por 16 dias más de suspendida aquélla.

En el transcurso de este período. a contar desde la mejoria clínica y del líquido céfalo-raquídeo, se practicaron 5 exámenes de líquido céfalo-raquídeo, siendo todos normales. Creemos justificado, por lo tanto, incluirlo dentro de los mejorados a meningitis a Pfeiffer. a pesar de que 8 días después de suspendida toda la terapia, y cuando estaba próxima al alta, presenta alza térmica de 40 grados, reaparecen los signos meníngeos, el líquido céfalo-raquideo se hace purulento y fallece al cabo de 4 dias, comprobándose en el líquido céfalo-raquídeo desarrollo de neumobacilo de Friedlander. Por todas estas consideraciones extamos autorizados a pensar que este úlitimo episodio fué independiente de la meningitis que motivó su hospitalización; y que probablemente la intensidad $d_{\bar{e}}$ la terapia intratecal ha creado un locus de menor resistencia, que facilitó el anidamiento de una nueva infección.

De los otros 2 cascs curados, uno (caso No 2) todavía permanece en el Hospital por razones ajenas a su meningitis: lleva alrededor de 6 meses de observación y está en espléndidas condicioses generales, sin presentar ninguna secuela hasta el momento. El otro caso (caso $N^{\circ} 1$ ) ha sido controlado un mes y medio después de haber sido dada de alta y está sana $y$ sin secuelas.

De los muertos, el caso $\mathrm{N}^{\circ} 4$ corresponde a un lactante de 6 meses que ingresa en estado de suma gravedad, con ataques convulsivos a repetición y que fallece al cabo de 22 días. Se trataba del primer enfermo de meningitis a Pfeiffer tratado con la asociación sulfa-penicilina y carecíamos de experiencia al respecto, siendo tratado, al parecer, en forma insuficiente. En efecto, en este enfermo, pese a la gravedad del cuadro, usamos la penicilina intra-raquídea en dosis inferiores a la mayoría de los otros casos $(10.000 \mathrm{U}$. diarias) y no la: 
ascciamos al tratamiento por vía intramuscular. Además. diante los 6 primeros días se usó dosis de sulfa relativamente bajas $(0,20 \mathrm{gr}$. por kilo de peso). El germen demostró una gran rebeldía al tratamiento, encontrándose en 6 exámenes sucesivos de líquido céfalo-raquídeo, lo que tampoco aconteció en los otros enfermos.

Mucho más interesante es el otro enfermo fallecido (Caso $N^{\text {to }} 5$ ), en cuya evolución pudo comprobarse en forma precisa la acción de la penicilina sobre el cuadro meningeo y el líquido céfalo-raquídeo. En efecto, al cabo de 11 días de terapia combinada, este enfermo manifiesta una franca mejoría, a ta! punto que nos atrevemos a suspender la penicilina, ya que el líquido céfalo-raquideo era claro y contenía 0.40 gr. de glucosa $o / 00$. Permanece 3 días afebril, reapareciendo la fiebre, los signos meníngeos y el líquido céfalo-raquídeo se torna opalescente, con descenso de la glucosa a $0,10 \mathrm{gr}$. o/oo, reapareciendo el bacilo de Pfeiffer por segunda vez. Inmediatamente se reinstituye la penicilinoterapia y empieza a descender la temperatura, el riño está alegre, se alimenta bien, hay remisión de los signos meníngeos y el líquido céfalotaquideo se hace claro nuevamente a los 7 días. $E_{n}$ vista de la mejoría evidente experimentada por el enfermito, se vuelve a suspender la penicilina a los 5 dias de haber comprobado esta mejoria, para asistir nuevamente a una recaida, que chliga por tercera vez a implantar la terapia combinada. Se crintinúa durante 23 días en esta forma, período durante el cual el niño aparece como normal, y, en vista de que los últimos 3 líquidos céfalo-raquideos eran de aspecto transparente, acépticos, con albuminorraquia normal y con sólo ligeros descensos de la glucosa. y considerando que nuestro enfermito llevaba ya 43 punciones lumbares, suspendemos la terapia penicilínica, para esta vez reaparecer el cuadro meningeo en su máxima intensidad, que lo llev'a a la muerte al cabo de 83 días.

En síntesis: este niño presentó 3 recaidas, aparentemente relacionadas en forma directa con la suspensión del tratamiento penicilínico, to que nos induce a pensar que este enfermo pudo haber tenido resultado si se hubiera colocado dosis mayores de penicilina $y$ en forma continuada.

Alexander (2) señala que la meningitis a Pfeiffer, puede, en delerminadas circunstancias, ser tratada exclusivamente con sulfas con posibilidad de éxito, siempre que la primera muestra de líquido céfalo-raquídeo indique cifras de glucosa superiores a 0,40 gr. $\%$ oo. En nuestros enfermos, la glucosa 
del liquido céfalo-raquídeo extraido inmediatamente después del ingreso, revela cifras inferiores a $0,10 \mathrm{gr}$. $\% / 00$, con excepción de un caso (caso No 3), que era de $0.25 \mathrm{gr}$. $0 / 00$.

Es un principio general considerar en el pronóstico de una meningitis aguda purulenta, el momento en que se inicia la terapéutica, ya que como es lógico, mientras más precoz es éste, mayores posibilidades hay de éxito. Nuestros pacientes ingresaron al Hospital, cuando, según los datos anamnésicos, la afección probablemente se había iniciado entre 4 y 8 dias antes.

En este mismo orden de ideas y, como punto de referencia pronśstica, tiene importancia el estado de gravedad que el chinico observa y la terapia que estos enfermos hayan recibido antes de su ingreso. A este respecto, todos los niños fueron catalcgados en recepción como graves y 3 habían recibids tratamiento a base de sulfa, que puede suponerse de insuficiente o mal llevado, y 2 no habian recibido ningún tratamiento.

Er resumen. pensamos que las observaciones a que nos hemos referido, pertenecen a lactantes, que. en general, tenian las mayores pesibilidades de fallecer frente a una infección tan grave, y por lo tanto, estamos tentados de suponer que la asociación de la penicilina intra-raquidea con la sulfamidoterapia, podría explicar los resultados favorables en los casos mejorados.

Boraba $y$ colaboradores $(4,5,6,7$ y 8$)$, en Uruguay, han sido los primercs en dar a conocer en forma sucesiva numerosos casos de niños con meningitis a Pfeiffer curados con el tratamiento sulfa-penicilina, y si bien es cierto que la mayoría son mayores de 14 meses. los resultados finales $y$ las saracterísticas de la evolución clínica les ha permitido a estos autores preconizar el tratamiento combinado con entusiasmo.

Bonaba, en efecto, señala la rápida mejoría observada inmediatamente de iniciado el tratamiento con penicilina: a su vez, las recaidas, una vez cesado el tratamiento con ella, a pesar de haber continuado el tratamiento con sulfatiazol, $y$ concluye que la única conclusión prudente, por el momento, es que la asociación es evidentemente útil y que debe ser probada lo antes posible y continuada largo tiempo, a fin de ponerse a salvo de las posibilidades de agravación.

De acuerdo cen la experiencia recogida en 11 casos, de los cuales 8 curaron. Bonaba y colaboradores (7) recomiendan la terapéutica asociada, de modo de emplear la sulfamidoterapia a razón de $0,20 \mathrm{gr}$. por kilo de peso, intravenosa, y a 
ruzón de $0,40 \mathrm{gr}$. por kilo de peso, por vía oral, además de 20-30,000 U. de penicilina intra-raquídea cada 12 horas. dosis que deberá disminuirse a la mitad apenas se note alguna mejoría del enfermito, continuando en esta forma por 10 días, al cabo de los cuales se volverá a la dosis alta inicial, la que se sostendrá por 6 días, período para ellos el más crítico y en el cual suelen observarse la mayoría de las recaídas.

Schweitzer, en Argentina (17), haciéndose eco de 13 escuela uruguaya, refiere 4 observaciones de 2 niños de 16 meses de edad, curados con la asociación penicilina-sulfa. El sulfatiazol lo usó a dosis de $0,30 \mathrm{gr}$. por kilo de peso y por vía oral. En todos los casos usó la penicilina a razón de 20.000 U. diarias, colocadas en dos sesiones. También refiere ia curación de otros 2 casos de su clientela particular, uno de 4 y otro de 5 meses de edad, ambos tratados en la forma yo relatada.

¿Es que la penicilinoterapia por via raquídea ha influído realmente en ia curación de los enfermos relatados por los antores uruguayos, argentinos y en nuestros propios casos?

Si dejamos de considerar por un momento que todos estos enfermos han mejorado, sea porque en ellos habria bastado la sulfamidoterapia, sea porque se ha estado en presencia de una infección a gérmenes menos patógenos que lo habitaal, es interesante señalar algunos hechos clínicos y experimentales, que permitirían sostener que la penicilina puede ser un arma terapéutica más en las infecciones a Pfeiffer. En efecto. en estos últimos tiempos han venido apareciendo publicaciones en las cuales se sostiene que gérmenes del grupo de los bacilos Gram (- ) , hasta entonces considerados como resistentes a la penicilina, son sensibles cuando se los coloca frente a dosis más elevadas o cuando se aprovecha la acción sinérgica de los sulfamidos. Así. Ory y colaboradores (16) relatan un case de empiema a bacilo Gram (-), tratado exclusivamento con penicilina intrapleural y obtienen la curación completa. Estudian las mínimas concentraciones que producen la completa inbibición del germen in vitro frente a la penicilina $g$ y $x$ y también frente a la estreptomicina, demostrando que era 167 veces más sensible a la penicilina g que a la estreptomicina. Inyectando $200,000 \mathrm{U}$. de penicilina intrapleural cada 24 horas, se mostraron niveles de penicilina en el liquido pleural a las 18 y 24 horas, suficientes en exceso para la seasibilidad del microorganismo. 
Gatdner (12) demostró completa inhibición del bacilo tífico y también de la escherichia coli, y cuando se usaron concentraciones de penicilina de $5 \mathrm{U}$. por cc., fué posible observar la lisis completa del bacilo de Eberth. Posteriormente a estas observaciones, muchos otros investigadores han demostrado inhibición parcial o completa de bacilos Gram (-) por ia penicilina. Las concentraciones necesarias para producir este efecto en la mayoría de los bacilos Gram (-) fluctúa entre 50 y 5,000 veces de aquélla necesaria para la inhibición de $]_{\mathrm{a}}$ cepa standard oxford del estafilococus aureus. Por otra parte, Forgacs y colaboradores (11), estudiando la sensibilidad de varios gérmenes patógenos, y entre ellos el hemophilus influenzae, encuentran cepas que tienen idéntica sensibilidad a la mayoría de las cepas de estafilecocus aureus, obtenidos de casos clínicos.

La sensibilidad o la resistencia de ciertos gérmenes. entonces, es cuestión de concentraciones y su aplicación clínic? dependetá evidentemente de si los niveles obtenidos en los fluidos pueden elevarse a cifras que inhiban el desarrollo de los gérmenes aparentemente resistentes.

Sabemos que existen varios métodos para obtener niveles más altos de droga, sea usando dosis mayores, sea atrasando la absorción del medicamento, atrasando la excreción urinaria o haciendo aplicaciones locales en altas concentraciones en el sitio de la infección.

Importaría, en nuestro caso, en primer lugar, conocer a qứ dosis sería posible inhibir el desarrollo del $\mathrm{H}$. Influenzae, Y un segundo lugar, qué dosis de penicilina intra-raquídea y a gué ritmo debiera usarse. Con respecto al primer punto, Gordon (13) ha dado a conocer recientemente una serie de experiencias tendientes a demostrar que el $\mathrm{H}$. Influenzae es un germen susceptible de ser inhibido con la penicilina. En efetc. estudia 43 cepas provenientes del árbol respiratorio, demostrando que 31 de ellas eran completamente inhibidas con $2.5 \mathrm{U}$. de penicilina por $\mathfrak{c}$. de medio, y aun, en una concentración de l U. por cc. de medio, los crecinientos de sólo 1] cepas fueron completos y en 18 hubo marcada inhibición del desarrollo. Sólo 7 cepas mostraron crecimiento con $5 \mathrm{U}$. de fenicilina por $c c$ de medio, aunque éste fué marcadamente inhibido.

En 18 cepas aisladas de líquidos céfalo-raquídeos provenientes de enfermos con meningitis a Hemophilus Influenzae, ohtuvo en 16 de ellas completa inhibición del desarrollo con 
2,5 U. de penicilina, notándose total inhibición del crecimiento de 3 cepas aun con $0.5 \mathrm{U}$. de penicilina por cc. de medio.

Este interesante trabajo nos aporta el dato concreto que el Hemophilus Influenzae es susceptible de ser inhibido en su desarrollo cuando se obtienen niveles, en general superiores a 2,5 U. de penicilina por $c c$. de medio.

En cuanto al segundo problema, o sea, conocer qué cantidad de penicilina se necesita inyectar, y a qué ritmo para obtener el nivel deseado, vale la pena citar a Cooke y Goldring (10), quienes, en un grupo de 7 lactantes de 2,5 meses a 16 meses de edad, con meningitis aguda a diferentes gérmenes, han hecho observaciones diarias de los niveles de penicilina en el líquido céfalo-raquídeo 24 horas aproximadamente después de la inyección de penicilina intratecal. Todos los niños recibieron $10.000 \mathrm{U}$. de penicilina intraraquídea uns vez al día, conjuntamente con penicilina intramuscular en cantidades de $10,000 \mathrm{U}$. cada 3 horas, observándose quue el nivel de penicilina en el líquido céfalo-raquídeo permanecía relativamente constante en el mismo niño durante el período que se mantenía la terapia. Este nivel era más alto. alcanzando hasta 1-2 U. por cc. en los niños menores, que habian recibido, relativamente mayores dosis de penicilina intratecal, siendo más bajo $(0,2-0,4$ por $\mathrm{cc}$.) en los niños más grandes.

Basados en estas informaciones se podría suponer que la curación de los enfermos con la combinación sulfa-penicilina se debió a la obtención de niveles elevados de penicilina en el líquido céfalo-raquídeo y a la acción sinérgica de las sulfas. Es más probable que la inyección intra-tecal de 20,000 U. cada 8-12 boras esterilice el líquido céfalo-raquideo y permita obtener resultados halagadores cuando la meningitis sea de reciente iniciación y no haya tabicamiento.

Cooke.y Goldring observaron, además. que la penicilina puede persistir en el líquido céfalo-raquídeo hasta 72 horas después de suspendidas las inyecciones intra-tecales. También observaron que los niveles de penicilina en el líquido céfaloraquídeo eran ligeramente más altos después de varios días de estar administrándola, que a continuación de las dosis iniciales.

De esto se desprende que es necesario determinar, en general, cuando se está en presencia de una infección a germen Gram (-), su sensibilidad a la penicilina y usar ésta soła- 
mante si el microorganismo es sensible a las concentraciones. que el antibiótico pueda ser mantenido en el sitio de la infección. Es necesario tener presente que los gérmenes Gram (...) probablemente también son capaces de producir sustancias ina:tivadoras de la penicilina, lo cual debe ser tenido en cuenta para el dosaje y frecuencia de las inyecciones.

Es evidente que todos estos hallazgos e informaciones scn muy interesantes, ya que la penicilina pasa a cubrir en su aspecto terapéutico muchas otras afecciones, en las cuales se daba por descontada su ineficacia.

Con el advenimiento de la estreptomicina, sin embargo, es muy probable que estas nuevas indicaciones de la penicilina sean sustituídas en gran parte por aquélla. Las investigaciones filturas en este campo darán la última palabra, de si aun con el uso de la estreptomicina, algunas cepas que sean resistentes a esta última puedan ser sensibles a la penicilina. como en el caco de Ory y colaboradores.

Trabajos recientes $(3,15$ y 9 ) señalan el alto valor terapíutico de la estreptomicina en las infecciones a Hemophilus Influenzae, de modo que por el momento aparece cono la drega lógica en el tratamiento de estas afecciones.

\section{Resumen.}

Se dan a conocer 5 observaciones de meningitis a Pfeiffer en niños menores de 10 meses, tratados con la asociación sulfa-penicina, de los cuales 2 fallecieron y 3 curaron.

2. Se señala la penicilino-sensibilidad del Hemophilus Influenzae culando se pone in vitro frente a altas concentraciones de penicilina.

3. Se sugiere que bajo la acción sinérgica con la sulfonamida. probablemente niveles relativamente elevados en el liquido céfalo-raquídeo actúen destruyendo el bacilo de Pfeiffer.

4. Mientras no tengamos estreptomicina disponible, se aconseja estudiar la sensibilidad a la penicilina del germen causal y según ello emplear el tratamiento combinado penicilina-sulfa. 


\section{Bibliografía.}

1.--ALEXANDER, H. E.; ELLls, C. and LEIDY, G. - J. of Pediat. 20 : $673, .1942$.

2.-ALEXANiDER, H. E. - J. of Pediat. 25: 517, 1944.

3.--ALEXANDER, H. E. - J. of Pediat. 29 : 192, 1946.

4.-BONABA, J.; SURRACO; FORTLLO y SCOLPINI - Arch. Pediat. del Uruguay, 15: 653, 1944.

5.-BONABA, 3.: GIAMPIETRO y VIDAL. - Arch. Pediat. del Uruguay. 16: $737,1945$.

6.-BONABA, J.; SURRACO; CARITAT; SOLOVEY y FONSECA. Are. Pediat. del Uruguay. $16: 137,1945$.

7.-BONMBA, J. - - Ateb. Pediat, de! Uraguay, 17: 321, 1946.

8.-BONABA, J.; NEGRO, R. - Arch. Pediat. del Urugaay, 17: 40\%, 1946.

9,--BIRMINGHAM, M. D.; KAYE, R, and SMITH. M. H. D. - J. of Pediat. 29: 1, 1946.

10-COCKR, V. J.; GOLDRING, D. - J. A. M. A. 127; 80, 1945..

11.-FORGACS, P.; HUTCHINSON, R. L. and REWELL, R. E. - Lan. cet, 6356: 785, 1945.

12.-GARDNER, A. D. - Nature (London) 146: 837, 1940.

13.-GORDON, M. and ZINNEMANN, - Brit. M. J. 44.31: 795, 1945.

14.-MAYO DE SALVADOR; FIANTONI, C. Y TORRES, E. F, - A tch. Argentitios Pediat, 25: 339. 1946.

15.-NUSSBAUM, S.; GOODMAN, S.; ROBF'SON, C. and RAY, L. J. of. Pediaf. 29:14, 1946.

16.-ORY, E. M.; JACKSON, G. S, and FINLAND, M. - J. A. M. A. $131: 1035,1946$.

17;-SCHWEITZER. F. - Arch. Argentinos de Pediat., 17: 352, 1946. 